\title{
MedienPädagogik
}

Zeitschrift für Theorie und Praxis der Medienbildung

www.medienpaed.com

ISSN 1424-3636

Themenheft Nr. 37: Medienpädagogik als Schlüsseldisziplin in einer mediatisierten Welt. Perspektiven aus Theorie, Empirie und Praxis Herausgegeben von Henrike Friedrichs-Liesenkötter, Lara Gerhardts, Anna-Maria Kamin und Sonja Kröger

\section{Medienpädagogik in Theorie und Praxis weiterentwickeln}

\section{Der Beitrag der gestaltungs- und entwicklungsorientierten Bildungsforschung und der Grounded Theory}

Anna-Maria Kamin

\section{Zusammenfassung}

Der Beitrag greift die Diskussion um gegenstandsangemessene medienpädagogische Forschungsmethoden auf, die sowohl zur Theorie(weiter)entwicklung beitragen sollen als auch eine Qualitätsverbesserung der Praxis sichern. Die Etablierung von entwicklungs- und gestaltungsorientierten Forschungskonzepten, mit deren Hilfe die Forschung und Praxis systematisch miteinander verzahnt und sowohl forschungsbasierte Interventionen initiiert und begleitet als auch deren Wirksamkeit überprüft werden kann, scheint eine Lösung zu sein. Die Qualität solcher designbasierter Forschungen wird allerdings kontrovers diskutiert. Vor allem ein unzureichend eingelöster Anspruch an Güte und Generalisierbarkeit der Ergebnisse und dass der versprochene Beitrag der Theorieentwicklung vage bleibt, kann kritisiert werden. Mit einer Verzahnung der gestaltungs- und entwicklungsorientierten Bildungsforschung mit der Methodologie der Grounded Theory wird der Versuch unternommen, die Ansätze für die Weiterentwicklung von (medienpädagogischer) Theorie und Praxis zu fundieren. 


\title{
Further Development of Media Education in Research and Practice - The Contribution of Design- and Development- oriented Educational Research and Grounded Theory
}

\begin{abstract}
The article focuses on the discussion about appropriate media educational research methods, which increase the media educational theory development and thus the quality of media educational practice. A possible solution lies in the establishment of development- and design-oriented research concepts that systematically interlock with one another and are suitable to guide researchbased interventions and control their effectiveness simultaneously. However, the quality of such design-based research is controversial. Above all, the quality and generalizability of the results and a rather vague contribution to theory formation remain to be discussed. The tight interlocking of design- and development-oriented educational research with grounded theory methodology is an attempt to substantiate the further development of (media educational) theory and practice.
\end{abstract}

\section{Probleme traditioneller Forschungsrichtungen der Bildungswissenschaft}

In der Medienpädagogik wie auch in weiteren bildungswissenschaftlichen Disziplinen und in den Fachdidaktiken findet seit geraumer Zeit ein intensiver Diskurs über gegenstandsangemessene Forschungsverfahren statt. Grundlage ist die Kritik an den traditionellen bildungswissenschaftlichen Forschungsrichtungen wie der hermeneutischen und empirischen Forschung. Beiden wird eine mangelnde Kenntnis über die pädagogische Praxis selbst unterstellt. Bezogen auf die Medienpädagogik konstatieren Tulodziecki, Herzig und Grafe etwa «mangelnde Einsichten zum Zusammenhang von Bedingungen, innovativen Vorgehensweisen und Wirkungen in der medienpädagogischen Praxis» (Tulodziecki, Grafe, und Herzig 2013, 2). Als Ursache können Interessensunterschiede zwischen Bildungsforschung und Bildungspraxis angenommen werden. Schwerpunktmässiges Interesse der hermeneutischen Bildungsforschung ist die kulturelle 
Verständigung im argumentativen Diskurs; während die empirische Bildungsforschung vor allem auf die (möglichst) gesetzesmässige Erfassung und Abbildung von Zusammenhängen mittels empirischer Verfahren zielt (Reinmann und Sesink 2011, 39). Pädagogische Intervention und Innovation - also die grundlegenden Interessen der Bildungspraxis - stehen zumeist nicht primär im Fokus traditioneller hermeneutischer und empirischer Bildungsforschung. Da für die Bildungswissenschaft der Anspruch formuliert wird, die Praxis nicht nur abzubilden, «sondern [...] deren Sinnbestimmungen (Selbstbestimmung, Autonomie, Mündigkeit) mit ihr zu teilen und zu befördern (Herv. A.-M. K.)» (Reinmann und Sesink 2014, 77), kann hier bereits eine Problematik konstatiert werden. Als Zielstellung bildungswissenschaftlicher Forschung lässt sich neben der Beschreibung auch die Veränderung von Praxis in Form von Unterstützung bzw. Begleitung bei der Umsetzung von Entwürfen oder Konzepten in der Bildungspraxis festgehalten. Bildungswissenschaft als rein hermeneutische und empirische Disziplin kommt diesem Anspruch nicht hinreichend nach, sodass von einem mangelnden Realisierungsbezug (ebd.) gesprochen werden kann.

Ein weiteres Problem besteht in einem unzureichenden Zukunftsbezug beider Forschungsrichtungen. Hermeneutische wie auch empirische Forschung ist auf die Beschreibung/Erfassung der Vergangenheit oder der Gegenwart ausgerichtet. Daraus resultiert, dass die gewonnen Erkenntnisse nur begrenzt für die Bildungspraxis von Nutzen sind (vgl. ausführlicher in: Reinmann und Sesink 2014). Zwar werden aus den Erkenntnissen bildungswissenschaftlicher Forschung oftmals Empfehlungen für die Praxis abgeleitet; die Umsetzung der Empfehlungen sowie deren Überprüfung sind dabei in der Regel jedoch nicht Bestandteil des Forschungsverfahrens. Diese erfolgen - sofern vorgesehen - im Rahmen von Evaluationen. Evaluationen dienen hingegen lediglich der Überprüfung und ggf. Optimierung des Produktes/Konzeptes (Reinmann 2005, 36), wobei wiederum in der Regel empirische Verfahren eingesetzt werden. Es besteht insofern ein Bedarf der stärkeren Verzahnung von Theorie und (Bildungs-)Praxis, um eine engere Zusammenarbeit von Wissenschaft und Praxis in Form von «forschungsmethodische[n] Verknüpfungsmöglichkeiten zwischen beiden Systemen» (Niesyto 2014, 174) zu erzielen. Knaus, Meister und Tulodziecki fordern ferner 
«dass das Theorie-Praxis-Verhältnis [Herv. i. O.] als situationsbezogenes In-Beziehung-Setzen von vorhandenen Vorstellungen bei Praktikerinnen und Praktikern mit wissenschaftlichen Theorien und empirischen Ergebnissen zu konzipieren ist» (Knaus, Meister, und Tulodziecki 2018, 33).

\section{Design-, gestaltungs- und entwicklungsorientierte For- schungsansätze}

In der Weiterentwicklung früherer erziehungswissenschaftlicher Forschungsansätze und unter Bezugnahme auf die Postulate der DesignBased-Research (DBRC 2003) sind in den letzten 15 Jahren Konzepte entstanden, die den Dialog zwischen Bildungsforschung und Bildungspraxis forcieren und das Vorgehen in einem Forschungskonzept systematisieren.

In Bezug auf die Medienpädagogik handelt es sich dabei um die gestaltungsorientierte Bildungsforschung (Tulodziecki, Grafe, und Herzig 2013; 2014), die gestaltungsorientierte Mediendidaktik (Preußler, Kerres, und Schiefner-Rohs 2014) sowie die entwicklungsorientierte Bildungsforschung (Reinmann und Sesink 2014). Auch in weiteren Disziplinen der Bildungs- und Unterrichtsforschung sowie in den Fachdidaktiken wurden designorientierte Ansätze aufgegriffen und ausdifferenziert (z.B. Educational Design Research: van den Akker u.a. 2011; Design Research: Edelson 2002: Plomb und Nieveen 2007; Didactical Design Research bzw. Fachdidaktische Entwicklungsforschung: Prediger u.a. 2012).

Sämtliche Ansätze lassen sich über ihren gemeinsamen Grundgedanken miteinander verknüpfen, Entwicklung und Gestaltung als wesentliche Strukturebenen zu betrachten und insofern unmittelbare Ansatzpunkte für Planung und Veränderung zu bieten. Gestaltungs- bzw. Entwicklungsprozesse und Theorieentwicklung werden im Prozess der Forschung aktiv aufeinander bezogen (Edelson 2002, 107). Gestaltung bzw. Entwicklung werden insofern als Teil der Forschung verstanden, die im Dialog und in Interaktion mit den beteiligten Akteuren aus der Praxis systematisch durchgeführt und reflektiert wird sowie offen für Veränderungen bleiben soll. Theorie, Modellierung von Praxis, hermeneutische Verständigung, empirische Erprobung, Evaluation und theoriegeleitete Reflexion werden 
in Verbindung gebracht und als voneinander abhängig betrachtet. So ist es möglich, sowohl Erkenntnisse über die bestehende Realität als auch über die Erschliessung von Veränderungspotenzialen zu gewinnen (Reinmann und Sesink 2014, 81). Zugleich eröffnen sich «vielfältige Chancen für die forschungsbezogene Zusammenarbeit von Praktiker/innen und Wissenschaftler/innen» (Niesyto 2014, 176) indem «[i]m Unterschied zu TopDown-Entwicklungsprojekten [...] auf eine dialogische und partizipative Forschungspraxis gesetzt [wird]»(ebd.). Forschung erfolgt stets zyklisch und in Zusammenarbeit mit den Akteuren der Praxis.

Als geeignete Bereiche, in denen designbasierte Forschung eingesetzt werden kann, hat das Design-Based Research Collective (DBRC 2003, 8) die Mitglieder des DBRC, die den DBR-Ansatz für die Bildungsforschung fruchtbar gemacht haben - u.a. die Erkundung von Möglichkeiten für neuartige Lern-/Lehrarrangements, die Entwicklung kontextualisierter Theorien über Lehren und Lernen sowie die Sammlung von Erkenntnissen zur Konstruktion von Gestaltungsprinzipien vorgeschlagen.

Zur Umsetzung sind verschiedene phasenartige Konzepte entwickelt worden. Tulodziecki, Grafe und Herzig beschreiben für gestaltungsorientierte Forschungsvorhaben fünf Phasen, die sie jeweils in mehrere Forschungsschritte ausdifferenzieren (Tulodziecki, Grafe, und Herzig 2013, 203-4). Ausgehend von praktisch und theoretisch relevanten medienpädagogischen Fragen sind zunächst Kontextbedingungen zu klären und geeignete theoretische Grundlagen zu erarbeiten (1). Daraufhin sind ein theoriebasiertes Konzept für das praktische Handeln zu entwickeln (2) und konzeptbezogene Beispiele zu entwerfen (3). Die empirischen Untersuchungen zu den Beispielen sind nachfolgend zu planen und durchzuführen (4), abschliessend sind die empirisch ermittelten Ergebnisse einzuordnen und zu diskutieren (5) (Tulodziecki, Herzig, und Grafe 2014, 216). Reinmann und Sesink schlagen zur Umsetzung entsprechender Forschungsvorhaben fünf aufeinanderfolgende Phasen des Forschungsprozesses vor, deren Hintergrundfolie Dietrich Benners Strukturmodell erziehungswissenschaftlicher Forschung bildet (Reinmann und Sesink 2014, 87). Mit Blick auf die Konzeptionen von Tulodziecki, Grafe und Herzig sowie von Reinmann und Sesink lassen sich sieben Phasen beschreiben, die Grundlage für die weiterführenden Überlegungen sein sollen. 
Ausgangspunkt für die erste Phase, die Problematisierung, ist die Wahrnehmung, dass «etwas nicht stimmt» (Reinmann und Sesink 2014, 83). Handlungsleitend für die identifizierte Problemstellung sind dabei nicht (nur) normative Vorstellungen der Wissenschaft, sondern auch Probleme, die aus der konkreten Berufspraxis heraus entstehen. Reinmann und Sesink «plädieren für einen Diskurs zwischen Vertretern beider Instanzen im Rahmen einer Entwicklungspartnerschaft» (ebd., 84). Die zweite Phase stellt der Entwurf dar. Dieser hat den Anspruch, sowohl «den Erkenntnissen wie auch den Gestaltungsinteressen der Praxis» (ebd.) gerecht zu werden. Den Postulaten der entwicklungs- und gestaltungsorientierten Forschung folgend, bedeutet das, dass auch eine diskursive Verständigung über Ziele und Grundauffassungen zum Lernen zwischen Praktikern und Forschern anzustreben ist (Tulodziecki, Herzig, und Grafe 2014, 225). Die dritte Phase bezeichnen Reinmann und Sesink etwas missverständlich als «Experiment», obwohl sich das Forschungskonzept explizit von experimenteller Forschung abheben soll (Reinmann 2005, 63; siehe auch den Schritt 4 bei Tulodziecki, Herzig, und Grafe 2014, 221 und Tulodziecki, Herzig, und Grafe 2014, 4ff., sowie ausführlicher: Tulodziecki, Grafe, und Herzig 2013, 36ff.). Kamin und Meister (2017, 217) schlagen für diesen Schritt aus diesem Grund den Begriff «Erprobung» vor, in der das entworfene Szenario in der Praxis angewendet wird. Im vierten Schritt, der Auswertung, geht es um die Beurteilung der in der Erprobungsphase durchgeführten Massnahmen. Hierbei gilt es zu überprüfen, inwiefern der Entwurf umgesetzt werden konnte und welche Modifikationen gegebenenfalls notwendig wurden. Bei der Anwendung von Auswertungsmethoden besteht in der gestaltungs- und entwicklungsorientierten Forschung Einigkeit darüber, dass die Anwendung eines Methodenmixes aus klassischen Instrumenten der empirischen Sozialforschung wie Fragebogen, Beobachtungen, Interviews oder Dokumentenanalysen, also die Kombination aus qualitativen und quantitativen Verfahren, sinnvoll ist (Tulodziecki, Herzig, und Grafe 2014, 14; siehe auch: Preußler, Kerres, und Schiefner-Rohs 2014, 266). In der nachfolgenden fünften Phase des modifizierten Entwurfs geht es darum, den gewonnenen «Erkenntnisfortschritt» (Reinmann und Sesink 2014, 86) in einen neuerlichen Entwurf münden zu lassen, womit der Gestaltungs- und Entwicklungszyklus sich fortsetzt. Gestaltungs- und 
entwicklungsorientierte Forschung ist insofern prinzipiell unabgeschlossen. «Es müssen folglich von allen Beteiligten gemeinsam andere Kriterien für das vorläufige Ende eines Forschungsprozesses festgelegt werden» (ebd., 85) Ergänzend zu den fünf Phasen regen Reinmann und Sesink noch eine forschungsmethodische Reflexion und die Entwicklung von theoretischen Perspektiven an, die als Forschungsschritte hinzugefügt werden können (Kamin und Meister 2017, 216), womit gleichzeitig die von Tulodziecki, Grafe und Herzig geforderte Einordnung und Diskussion der empirisch ermittelten Ergebnisse berücksichtigt werden kann.

Die Begründungslinien für diese Forschungsrichtung werden in der medienpädagogischen Community bereits intensiv diskutiert, wie z.B. im Jahrbuch Medienpädagogik 10 «Methodologie und Methoden medienpädagogischer Forschung» (Hartung u.a. 2014), in dem von Gabi Reinmann laufend aktualisierten Reader mit Beiträgen zum Thema entwicklungsorientierte Bildungsforschung (Reinmann 2017a) oder in Beiträgen von Tulodziecki, Herzig und Grafe (z.B. 2013; 2014). In einer Vielzahl von Forschungsarbeiten im Kontext der Medienpädagogik wurden die Ansätze bereits aufgegriffen, erprobt und weiterentwickelt (z.B. Koppel 2017; Kamin und Meister 2017; Martin 2018) und stossen sowohl in der Forschung als auch in der Praxis auf grosse Akzeptanz. So fordert die Gesellschaft für Medienpädagogik und Kommunikationskultur - GMK e.V. (Knaus, Meister, und Tulodziecki 2018, 37) als grösster medienpädagogischer Berufsverband mit Mitgliedern aus Wissenschaft und Praxis in einem Positionspapier zur Qualitätsentwicklung: «Die Medienpädagogik sollte Bemühungen um eine gestaltungsorientierte Forschung verstärken, in der praxis- und theoriegeleitete Entwicklung von Konzepten für medienpädagogisches Handeln und deren empirische Evaluation zentral sind» (Knaus, Meister, und Tulodziecki 2018, 37). Inzwischen wird gar diskutiert, ob die Forschungsrichtung auf dem Weg ist, sich als Mainstreamforschung zu etablieren (Reinmann 2017b).

Dennoch bleibt festzuhalten, dass weiterhin Entwicklungsbedarf besteht. Tulodziecki, Grafe und Herzig (2014, 215) weisen darauf hin, «dass allgemeine Standards für die methodische Durchführung und die Dokumentation sowie für die Bewertung von Ergebnissen noch ausstehen» wenn sie auch zehn Qualitätsanforderungen für die gestaltungsorientierte 
Forschung herausarbeiten $(2013,235)$. Gestaltungsorientierte Bildungsforschung steht insofern vor der Herausforderung, sich über ihre Methoden zu verständigen sowie einen Konsens über methodisch angemessene Vorgehensweisen zu erzielen (Preußler, Kerres, und Schiefner-Rohs 2014, 257). Insbesondere bedürfen das Verhältnis von Theorie und Kontextfaktoren sowie Fragen bzgl. der Generalisierung bzw. Übertragbarkeit einer Konkretisierung (Tulodziecki, Grafe, und Herzig 2014, 215). Kamin und Meister $(2017,222)$ schlagen diesbezüglich eine Verzahnung der gestaltungs- und entwicklungsorientierten Bildungsforschung mit der Methodologie der Grounded Theory vor und machen auf Entwicklungspotenziale aufmerksam (siehe auch Kamin 2013, 21; 2017, 338-39).

\section{Die Grounded Theory als Methode der Theorieentdeckung}

Um die Verknüpfungs- und Entwicklungspotenziale der gestaltungs- und entwicklungsorientierten Bildungsforschung mit der Grounded Theory darzustellen, gilt es zunächst, die Postulate der Methodologie zu skizzieren. Es handelt sich um ein qualitatives Forschungsverfahren, welches das Ziel verfolgt, eine induktiv hergeleitete, gegenstandsbezogene Theorie eng am erhobenen Material arbeitend zu generieren bzw. zu entdecken (Strauss und Corbin 1996, 8). Ziel der Vorgehensweise ist, die Bedeutung menschlicher Erfahrungen zur Aufdeckung sozialer Strukturen im Prozess praktischer Forschung offenzulegen (Corbin 2006, 70). Intention der Protagonisten war es u.a., für die Praxis zu forschen, um z.B. Leitlinien für professionelles pädagogisches Agieren zu entwickeln (Aßmann 2013, 82). Das Forschungsverständnis der Grounded Theory lässt sich anhand von fünf Grundprinzipien verdichten:

- Wechselprozess von Datenerhebung, Auswertung und Theoretischem Sampling;

- Theorieorientiertes Kodieren;

- Ständiges Vergleichen;

- Schreiben theoretischer Memos sowie;

- Relationierung von Datenerhebung, Kodieren und Memoschreiben im gesamten Forschungsprozess (Przyborski und Wohlrab-Sahr 2009, 194-203). 
Im Vergleich zu traditionellen Forschungsdesigns, die durch eine lineare Abfolge von Forschungsschritten gekennzeichnet sind, geht die Grounded Theory-Methodologie von einer Interdependenz von Theorie und Empirie aus. «Diesem Konzept zufolge wechseln sich die Auswahl erster Fälle aufgrund einer relativ offenen sozialwissenschaftlichen Fragestellung, Interpretation, erste Hypothesenbildung, erneute Fallauswahl und fortschreitende Theorieentwicklung ab» (Przyborski und Wohlrab-Sahr 2009, 177). ${ }^{1}$ Da sich empirische Beobachtungen aber nicht von theoretischen Einflüssen befreien lassen (Kelle 2011, 236), sprechen Glaser und Strauss von Sensibilisierenden Konzepten, die als Leitideen den Ausgangpunkt von Forschungen darstellen (Böhm 2000, 476). Quellen theoretischer Sensibilität können neben vorgängigen Forschungserfahrungen auch Berufs- und individuelle Erfahrungen sein; ebenso kann Fachliteratur die theoretische Sensibilität anregen (Strauss und Corbin 1996, 25-26; Mey und Mruck 2011, 31). Diese Konzepte stützen die empirischen Ergebnisse und werden zur Erklärung der Phänomene herangezogen, d. h. sie verleihen den Daten Bedeutung (Strauss und Corbin 1996, 56). Sie bilden insofern nicht nur den Ausgangspunkt der Forschung, sondern werden auch zur Interpretation der Daten herangezogen.

Die Methodologie der Grounded Theory sieht parallel zum Auswertungsprozess das Anfügen von Memos in Form von theoretischen Konzepten vor.

«Neue Theorien können nicht allein aufgrund von empirischen Daten ab ovo entwickelt werden, sondern erfordern eine Verknüpfung zwischen altem Theoriewissen und neuer empirischer Evidenz» (Kelle 2007, 47).

Eine solche Forschungspraxis mit der Bildung erklärender Hypothesen aus dem empirischen Datenmaterial sowie der Identifizierung relevanter theoretischer Konzepte wird als Abduktion bezeichnet (Strübing 2008, 45). Strübing betont, dass neue Erkenntnis sowohl zu einem Teil auf

1 Die konkreten Auswertungsschritte der Grounded Theory können hier lediglich angedeutet werden. Ergänzend ist auf den umfangreichen Fundus an Grundlagenliteratur hinzuweisen, etwa: Strauss und Corbin 1996; Strübing 2008; Mey und Mruck 2011; Best-Practices stellen aus Sicht der Verfasserin auch die Dissertationen von Aßmann (2013) und Steinhausen (2015) dar. 
Erfahrungen basieren kann als auch wesentlich aus spontanen Einfällen, «abduktiven Blitzen» (ebd., 50), gewonnen wird. Abduktion wird dabei nicht als exakte Methode oder ein Schritt für Schritt abzuschreitendes Verfahrensprogramm aufgefasst, das «herbeigezwungen» (Reichertz 2011, 287) werden könnte. «Die Abduktion ist ein mentaler Prozess, ein geistiger Akt, ein gedanklicher Sprung, der das zusammenbringt, von dem man nie dachte, dass es zusammengehört» (ebd., 286). Insofern ist

«[...] der abduktive Denkprozess [...] das Ergebnis einer Haltung gegenüber Daten und gegenüber dem eigenen Wissen: Daten sind ernst zu nehmen, und die Gültigkeit des bislang erarbeiteten Wissens ist einzuklammern» (ebd., 288).

Zusammenfassend kann konstatiert werden, dass die Grounded Theory-Methodologie ein iterativ-zyklisches Vorgehen kennzeichnet. Resultat dieser Wechselbeziehung ist ein abduktives Schlussfolgern, mithilfe dessen die Verknüpfung von empirisch emergierenden Ergebnissen und theoretischen Erkenntnissen erfolgt. Dabei ist zu beachten, dass sich die Grounded Theory-Methodologie nicht nur als Sammlung einzelner $\mathrm{Me}$ thodenelemente versteht, «[...] sondern als spezifischer Forschungsstil, der sich deutlich von jenem traditionellen sequenziellen Vorgehen unterscheidet» (Mey und Mruck 2011, 23). Das bedeutet, dass der wechselseitige Prozess von Datenerhebung und Datenanalyse und nicht die einzelnen Auswertungsschritte in den Vordergrund gestellt werden.

Es stellt sich die Frage, inwieweit die Methodologie der Grounded Theory hilfreich bei der Weiterentwicklung der gestaltungs- und entwicklungsorientierten Forschung sein kann. Es gilt zu diskutieren, inwiefern eine Verschränkung dazu beitragen kann, den Aspekt der Theoriebildung stärker zu betonen und methodisch zu fundieren. Weiterhin ist zu klären, ob die Grounded Theory zur Erarbeitung von verbindlicheren Standards von gestaltungs- und entwicklungsorientierter Forschung beitragen kann. 


\section{Gestaltungs- und entwicklungsorientierte Bildungsfor- schung und Grounded Theory - Verknüpfungspotenziale ${ }^{2}$}

Im Hinblick auf mögliche Verschränkungen zwischen gestaltungs- und entwicklungsorientierter Bildungsforschung und der Grounded Theory sind zunächst Gemeinsamkeiten im Hinblick auf Zielvorstellungen zu identifizieren, um daraufhin einen Vorschlag zu erarbeiten wie (a) ein mögliches Verhältnis konzeptionell zu fassen ist und (b) Grundprinzipien und Forschungsschritte in einzelne Phasen der gestaltungs- und entwicklungsorientierten Forschung Eingang finden können, um Verknüpfungspotenziale auch im Prozess der Forschung nutzbar zu machen.

Obwohl in der gestaltungs- und entwicklungsorientierten Forschung vor allem die Gestaltung von Praxis im Vordergrund steht und es in der Grounded Theory schwerpunktmässig um die Generierung von sozialwissenschaftlicher Theorie geht, lassen sich gemeinsame Zielvorstellungen erkennen. In der gestaltungs- und entwicklungsorientierten Bildungsforschung ist auch die Entwicklung von theoretischen Perspektiven intendiert und die Grounded Theory verfolgt ebenso den Anspruch auf Praxisrelevanz. Der Stellenwert von Theorie und Praxis im Forschungsprozess unterscheidet sich hingegen grundlegend. Während in Forschungsarbeiten, in denen die Grounded Theory angewendet wird, lediglich über und für die Praxis geforscht wird, forscht die gestaltungs- und entwicklungsorientierte Forschung auch mit der Praxis gemeinsam. Die gestaltungsund entwicklungsorientierte Forschung legt ihren Fokus auf Theoriefundierung und Theorieweiterentwicklung, der Grundgedanke der Grounded Theory ist die Theorieentdeckung (siehe dazu auch: Tulodziecki, Grafe, und Herzig 2014, 225; Tulodziecki, Herzig, und Grafe 2014, 12; ausführlicher: Tulodziecki, Grafe, und Herzig 2013, 24-27, 232).

Gemeinsamkeiten sind im Hinblick auf das Vorgehen im Forschungsprozess erkennbar. Ausgangspunkt der Forschung bzw. Forschungsanlass sind in beiden Konzepten eine Problemstellung aus Forschung und/oder Praxis. Eine weitere Gemeinsamkeit besteht in der Abfolge der jeweiligen Forschungsschritte. Sowohl in gestaltungs- und entwicklungsorientierten Forschungsansätzen als auch in der Grounded Theory ist das

2 Insbesondere in Bezug auf diesen Abschnitt danke ich Gerhard Tulodziecki und Silke Grafe für ihr konstruktives Feedback im Rahmen des Peer-Coaching-Prozesses für den Band. 
Vorgehen nicht linear, sondern iterativ-zyklisch. Die Iterationen in der gestaltungs- und entwicklungsorientierten Forschung beziehen sich auf die Gestaltungs- und Entwicklungszyklen, in denen ein kontinuierliches In-Bezug-Setzen von Theorie, Modellierung von Praxis, hermeneutischer Verständigung, empirische Erprobung, Evaluation und theoriegeleiteter Reflexion (s.o.) erfolgt. Das Vorgehen in der Grounded Theory ist sowohl im Hinblick auf die Samplingstrategie als auch auf den Prozess der Theoriebildung zyklisch. Das Sample wird nicht zu Beginn der Untersuchung festgelegt, «[...] sondern nach den theoretischen Gesichtspunkten, die sich im Verlauf der empirischen Analyse herauskristallisieren erst nach und nach zusammengestellt» (Przyborski und Wohlrab-Sahr 2009, 177). Daraufhin erfolgt - ausgehend von der Erhebung und Analyse des Datenmaterials durch verschiedene Stufen des Kodierens mithilfe der Sensibilisierenden Konzepte - die sukzessive Generierung von Theorieschnipseln (Mey und Mruck 2011, 15; Kamin 2013, 26), die sich mit fortschreitender Analyse zu einer Schlüsselkategorie abstrahieren lassen.

Im Hinblick auf das konzeptionelle Verhältnis (siehe a) könnte die Grounded Theory für die gestaltungs- und entwicklungsorientierte Bildungsforschung besonders gewinnbringend sein, wenn sich bei einer Problemstellung aus der Praxis zeigt, dass bisherige Theorien unter Umständen zwar Ansätze liefern, aber noch nicht hinreichend sind, um den Entwicklungs- und Gestaltungsanforderungen (ganz) gerecht zu werden und im Forschungsprozess neu entwickelt werden müssen.

Mit Blick auf mögliche Verknüpfungen mit den Forschungsschritten der gestaltungs- und entwicklungsorientierten Bildungsforschung (siehe b) ist denkbar, das methodische Vorgehen und die gut ausgearbeiteten Konzepte aus der Grounded Theory Methodologie für einzelne Phasen der gestaltungs- und entwicklungsorientierten Forschung fruchtbar zu machen.

Neben der Analyse von qualitativ-empirischen Daten in der Phase der Auswertung von gestaltungs- und entwicklungsorientierten Forschungsvorhaben nach den Auswertungsschritten der Grounded Theory lässt sich das Konzept der theoretischen Sensibilität für gestaltungs- und entwicklungsorientierte Forschungsvorhaben fruchtbar machen. Sensibilisierende Konzepte sind als heuristische Konzepte zur Identifizierung 
theoretisch relevanter Phänomene im Datenmaterial zu verstehen, die zusammengenommen den begrifflich-heuristischen Horizont und den Rahmen für das leitende Erkenntnisinteresse bilden (Kelle 2007, 38). Akteure aus Bildungsforschung und Bildungspraxis könnten insofern in der Phase der Problemstellung die formulierten Ausgangsideen der Forschung in Form von Sensibilisierenden Konzepten konkret gemeinsam identifizieren, sich darüber verständigen und dann systematisch dokumentieren. In der Phase der Auswertung können sie ferner gezielt zur Interpretation der Daten herangezogen werden (s.o.).

Die Entwicklung von theoretischen Perspektiven bleibt in der gestaltungs- und entwicklungsorientierten Bildungsforschung bislang teilweise vage. Die «schubweise» Entwicklung von Theorie als abduktives Schlussfolgern (Przyborski und Wohlrab-Sahr 2009, 196) verspricht Brauchbarkeit für die gestaltungs- und entwicklungsorientierte Bildungsforschung, um zur Entwicklung eines empirisch und theoretisch hergeleiteten und mit der Praxis abgestimmten Gesamtkonzepts beizutragen. Der wechselseitige Prozess von Induktion, Deduktion und Abduktion birgt allerdings das Problem, dass der Anschein der Beliebigkeit erweckt wird (Strübing 2008, 10). Dies gilt vor allem für den Prozess der Abduktion, weil dieser «geistig» (Reichertz 2011, 286) abläuft. Hier bietet sich die Phase der forschungsmethodischen Reflexion an, indem Forschungsschritte, Annahmen, und Vorgehensweisen transparent gemacht werden (siehe auch Kamin 2013, 27 und 2017, 337f.).

Insgesamt ist erkennbar, dass eine Verknüpfung von gestaltungs- und entwicklungsorientierten Forschungsansätzen mit der Grounded Theory gewinnbringend sein kann, sodass sowohl die Bildungsforschung als auch die Bildungspraxis profitieren können. Für Forschungsvorhaben ist insgesamt festzuhalten, dass keinesfalls ein rezeptartiges Vorgehen formuliert bzw. praktiziert werden sollte. Zu betonen ist darüber hinaus, dass gestaltungs- und entwicklungsorientierte Forschung ohne Grounded Theory keineswegs als defizitär zu betrachten ist. Fruchtbar kann vielmehr das konsequente Mit- und Zusammendenken der Ansätze im Prozess der Forschung sein, insbesondere wenn die Grounded Theory nicht nur als (Auswertungs-)Methode, sondern als Forschungsstil und Forschungshaltung verstanden wird (Mey und Mruck 2011, 22). Für die Medienpädagogik birgt 
die Verknüpfung die Chance, Aussagen aus handlungswissenschaftlicher Perspektive zu treffen, wie "gesellschaftliche sowie pädagogisch relevante Prozesse so ausgestaltbar sind, dass sie fördernd unterstützt werden.» (Pietraß 2018, 12; DGfE Sektion Medienpädagogik 2017, 2).

\section{Literatur}

Akker, Jan van den, Koeno Gravemeijer, Susan Mc Kenney, und Nienke Nieven, Hrsg. 2011. Educational Design Research. London: Routledge.

Aßmann, Sandra. 2013. Medienhandeln zwischen formalen und informellen Kontexten: Doing connectivity. Wiesbaden: Springer VS.

Böhm, Andreas. 2000. «Theoretisches Codieren: Textanalyse der Grounded Theory». In Qualitative Forschung: Ein Handbuch, herausgegeben von Uwe Flick, Ernst von Kardoff, und Ines Steinke, 475-85. Reinbek bei Hamburg: Rowohlt.

Corbin, Juliet. 2006. «Grounded Theory». In Hauptbegriffe qualitativer Sozialforschung, herausgegeben von Ralf Bohnsack, Winfried Marotzki, und Michael Meuser, 2., 70-75. UTB Erziehungswissenschaft, Sozialwissenschaft 8226. Opladen: Budrich.

DBRC. 2003. «Design Based Research: An Emerging Paradigmen für Educational Inquiry». Educational Researcher 32 (1): 5-8.

DGfE Sektion Medienpädagogik. 2017. «Orientierungsrahmen für die Entwicklung von Curricula für medienpädagogische Studiengänge und Studienanteile». MedienPädagogik: Zeitschrift für Theorie Und Praxis Der Medienbildung (Statements and Frameworks), 1-7. https://doi.org/10.21240/mpaed/00/2017.12.04.X.

Edelson, Daniel C. 2002. «Design Research: What We Learn When We Engage In Design». The Journal of the Learning Sciences 11 (1): 105-21. https://doi. org/10.1207/S15327809JLS1101_4.

Hartung, Anja, Bernd Schorb, Horst Niesyto, Heinz Moser, und Petra Grell, Hrsg. 2014. Methodologie und Methoden medienpädagogischer Forschung. Jahrbuch Medienpädagogik, 10: Research. Wiesbaden: Springer VS. https://doi. org/10.1007/978-3-658-04718-4.

Kamin, Anna-Maria. 2013. Beruflich Pflegende als Akteure in digital unterstützten Lernwelten: Empirische Rekonstruktion von berufsbiografischen Lernmustern. Wiesbaden: Springer Fachmedien Wiesbaden. https://doi.org/10.1007/978-3658-02310-2.

Kamin, Anna-Maria. 2017. "Lernen mit digitalen Medien Rekonstruktion empirisch emergierter und abduktiv generierter berufsbiografischer Lerngewohnheiten». In Forschungswerkstatt Medienpädagogik: Projekt - Theorie - Methode, herausgegeben von Thomas Knaus, 1:317-45. München: kopaed. 
Kamin, Anna-Maria, und Dorothee M. Meister. 2017. «Digital unterstütztes Lernen in den Pflegeberufen unter entgrenzten Bedingungen - ein gestaltungsund entwicklungsorientiertes Forschungsprojekt». In Jahrbuch Medienpädagogik 13: Vernetzt und entgrenzt - Gestaltung von Lernumgebungen mit digitalen Medien, herausgegeben von Kerstin Mayrberger, Johannes Fromme, Petra Grell, und Theo Hug, 213-29. Wiesbaden: Springer Fachmedien Wiesbaden. https://doi.org/10.1007/978-3-658-16432-4.

Kelle, Udo. 2007. "Theoretisches Vorwissen und Kategorienbildung in der 〈Grounded Theory〉》. In Qualitative Datenanalyse: computergestützt: Methodische Hintergründe und Beispiele aus der Forschungspraxis, herausgegeben von Udo Kuckartz und Heiko Grunenberg, 2., überarbeitete und erweiterte Aufl., 32-49. Wiesbaden: VS Verlag für Sozialwissenschaften/ GWV Fachverlage GmbH Wiesbaden.

Kelle, Udo. 2011. «(Emergence〉 oder 〈Forcing〉? Einige methodologische Überlegungen zu einem zentralen Problem der Grounded-Theory». In Grounded Theory Reader, herausgegeben von Günter Mey, 2., aktualisierte und erweiterte Aufl., 235-60. Wiesbaden: VS Verlag für Sozialwissenschaften.

Knaus, Thomas, Dorothee M. Meister, und Gerhard Tulodziecki. 2018. «Qualitätsentwicklung - Professionalisierung - Standards. Thesen aus medienpädagogischer Sicht». In Futurelab Medienpädagogik. Qualitätsentwicklung. Professionalisierung. Standards, 23-47. Schriften zur Medienpädagogik 45. München: kopaed.

Koppel, Ilka. 2017. Entwicklung einer Online-Diagnostik für die Alphabetisierung: Eine Design-Based Research-Studie. Wiesbaden: Springer Fachmedien Wiesbaden.

Martin, Alexander. 2018. Medienerziehung im Unterrichtsfach Pädagogik. Entwicklung und Evaluation einer Lehrkräftefortbildung zu den Themenbereichen $C_{Y}$ bermobbing, Gewalt und Sexualisierung in Medien. Bd. 650. Münster: Waxmann.

Mey, Günter, und Katja Mruck. 2011. «Grounded-Theory-Methodologie: Entwicklung, Stand, Perspektiven». In Grounded Theory Reader, herausgegeben von Günter Mey, 2., aktualisierte und erweiterte Aufl., 11-48. Wiesbaden: VS Verlag für Sozialwissenschaften.

Niesyto, Horst. 2014. «Medienpädagogische Praxisforschung». In Methodologie und Methoden medienpädagogischer Forschung., herausgegeben von Anja Hartung, Bernd Schorb, Horst Niesyto, Heinz Moser, und Petra Grell, 173-91. Jahrbuch Medienpädagogik, 10: Research. Wiesbaden: Springer VS. https://doi. org/10.1007/978-3-658-04718-4.

Pietraß, Manuela. 2018. «Die Ermöglichung von Lernen und Bildung im digitalen Raum. Medienpädagogische Perspektiven». In Jahrbuch Medienpädagogik 14. Der digitale Raum - Medienpädagogische Untersuchungen und Perspektiven, herausgegeben von Manuela Pietraß, Johannes Fromme, Petra Grell, und Theo Hug, 11-32. Wiesbaden: Springer Fachmedien Wiesbaden. https://doi. org/10.1007/978-3-658-19839-8_2. 
Plomb, Tjeerd, und Nienke Nieveen, Hrsg. 2007. An Introduction to Educational Design Research: Proceedings of the seminar conducted at the East China Normal University, Shanghai (PR China). 3. Aufl. Enschede: SLO. http://www.slo. nl/downloads/2009/Introduction_20to_20 education_2Odesign_2Oresearch. pdf/download.

Prediger, Susanne, Michael Link, Renata Hinz, Stephan Hußmann, Jörg Thiele, und Bernd Ralle. 2012. "Lehr-Lernprozesse initiieren und erforschen: Fachdidaktische Entwicklungsforschung im Dortmunder Modell». http://www.mathematik.uni-dortmund.de/ prediger/veroeff/12-Prediger_et_al_MNU_FUNKEN_Webversion.pdf.

Preußler, Annabell, Michael Kerres, und Mandy Schiefner-Rohs. 2014. "Gestaltungsorientierung in der Mediendidaktik: Methodologische Implikationen und Perspektiven». In Jahrbuch Medienpädagogik 10. Methodologie und Methoden medienpädagogischer Forschung., herausgegeben von Anja Hartung, Bernd Schorb, Horst Niesyto, Heinz Moser, und Petra Grell, 253-74. Wiesbaden: Springer VS. https://doi.org/10.1007/978-3-658-04718-4.

Przyborski, Aglaia, und Monika Wohlrab-Sahr. 2009. Qualitative Sozialforschung: Ein Arbeitsbuch. München: Oldenbourg.

Reichertz, Jo. 2011. «Abduktion: Die Logik der Entdeckung der Grounded Theory». In Grounded Theory Reader, herausgegeben von Günter Mey, 2., aktualisierte und erweiterte Aufl., 279-97. Wiesbaden: VS Verlag für Sozialwissenschaften.

Reinmann, Gabi. 2005. «Innovation ohne Forschung? Ein Plädoyer für den Design-Based Research-Ansatz in der Lehr-Lernforschung» Unterrichtswissenschaft (1): 52-69.

Reinmann, Gabi. 2017a. "Design-based research on the way to mainstream research? Comments on the plea for phronesis by Bardone and Bauters Educational Design», Research I (1), 1: 1-9.

Reinmann, Gabi. 2017b. Reader zum Thema entwicklungsorientierte Bildungsforschung. http://gabi-reinmann.de/?page_id=40.

Reinmann, Gabi, und Werner Sesink. 2011. «Entwicklungsorientierte Bildungsforschung: Diskussionspapier zur Herbsttagung der Kommission Medienpädagogik der DGfE». Leipzig. https://gabi-reinmann.de/wp-content/uploads/2011/11/Sesink-Reinmann_Entwicklungsforschung_v05_20_11_2011. pdf.

Reinmann, Gabi. 2014. «Begründungslinien für eine entwicklungsorientierte Bildungsforschung». In Jahrbuch Medienpädagogik 10. Methodologie und Methoden medienpädagogischer Forschung., herausgegeben von Anja Hartung, Bernd Schorb, Horst Niesyto, Heinz Moser, und Petra Grell, 75-89. Wiesbaden: Springer VS. https://doi.org/10.1007/978-3-658-04718-4.

Steinhausen, Julia. 2015. Berufsbiografische Zweigleisigkeit im Entscheidungsprozess zwischen Promotion und Referendariat. Universität Paderborn. https://nbnresolving.org/urn:nbn:de:hbz:466:2-17496.

Strauss, Anselm, und Juliet Corbin. 1996. Grounded theory: Grundlagen qualitativer Sozialforschung. Weinheim: Beltz PsychologieVerlagsUnion. 
Strübing, Jörg. 2008. Grounded Theory: Zur sozialtheoretischen und epistemologischen Fundierung des Verfahrens der empirisch begründeten Theoriebildung. 2., überarbeitete und erweiterte Aufl. Qualitative Sozialforschung 15. Wiesbaden: VS Verlag für Sozialwissenschaften. https://doi.org/10.1007/978-3-53191968-3.

Tulodziecki, Gerhard, Silke Grafe, und Bardo Herzig. 2013. Gestaltungsorientierte Bildungsforschung und Didaktik: Theorie - Empirie - Praxis. Bad Heilbrunn: Verlag Julius Klinkhardt.

Tulodziecki, Gerhard, Silke Grafe, und Bardo Herzig. 2014. «Praxis- und Theorieorientierte Entwicklung und Evaluation von Konzepten für medienpädagogisches Handeln als gestaltungsorientierte Bildungsforschung». In Methodologie und Methoden medienpädagogischer Forschung., herausgegeben von Anja Hartung, Bernd Schorb, Horst Niesyto, Heinz Moser, und Petra Grell, 213-29. Jahrbuch Medienpädagogik, 10: Research. Wiesbaden: Springer VS. https:// doi.org/10.1007/978-3-658-04718-4_11.

Tulodziecki, Gerhard, Bardo Herzig, und Silke Grafe. 2014. «Medienpädagogische Forschung als gestaltungsorientierte Forschung vor dem Hintergrund praxisund theorierelevanter Forschungsansätze in der Erziehungswissenschaft». MedienPädagogik: Zeitschrift für Theorie Und Praxis Der Medienbildung 2014 (Occasional Papers), 1-18. https://doi.org/10.21240/mpaed/00/2014.03.10.X. 